\title{
THE THERMAL ANALYSIS OF THE MUSHY ZONE AND GRAIN STRUCTURE CHANGES DURING DIRECTIONAL SOLIDIFICATION OF SUPERALLOYS
}

\author{
S. U. An, V. Larionov*, V. Monastyrski**, E. Monastyrskaia*, I. Grafas*, \\ J. M. Oh***, O. D. Lim, S. H. Kim, J. H. Lee****, D. Y. Seo*****

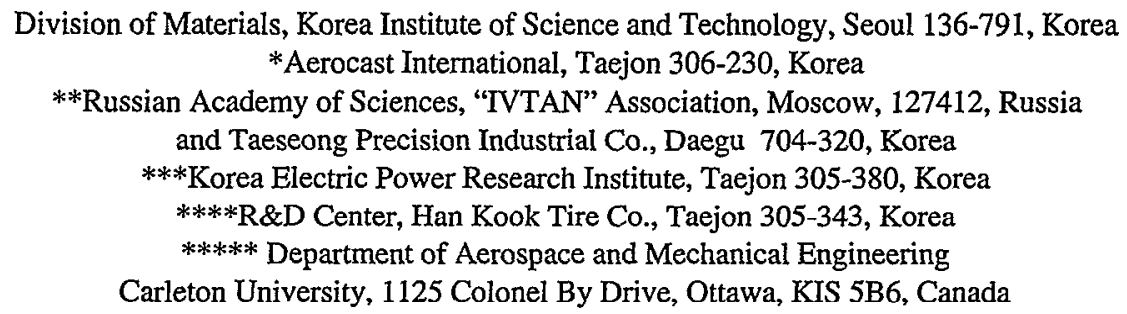

Abstract

The influence of the casting parameters (such as heater temperature, withdrawal rate and thermal conductivity of the mould) on the mushy zone of directionally solidified (DS) casting by the Bridgeman's method have been studied by computer simulation. The grain widening phenomena in DS castings on two superalloys IN738LC and B1914 has been investigated. Based on the geometrical theory of moving grain interface, an empirical dependence of the columnar grains widening on the distance from the nucleation site has been obtained.

\section{Introduction}

The advantages of using columnar structured buckets in newly designed high temperature engines manifests itself by increased efficiency, life cycle and decreased overall running cost. The elimination of transverse grain boundaries increases the creep strength and ductility of the material at operating temperatures and its resistance to relative thermal-cycle fatigue. The growth structure conditions provide high density of casting material by decreasing micro porosity and segregation, and changing the shape and distribution of carbides. All these factors influence the structural stability at high operating temperatures.

To define the growth conditions of casting structure during DS process the following parameters are used: temperature gradient, cooling rate and solidification rate. The dependence of their values on technical casting parameters has been extensively studied experimentally and theoretically. It is known, that the increase of heater temperature results in an increase of temperature gradient, and the increase of withdrawal rate results in an increase of melt cooling rate. The change of growth conditions is esteemed as a consequence of change of heat flows transferred through the mushy zone. During DS process of superalloys, the extension of the mushy zone along a direction of the solidification can be some centimeters. In this case a change of technical casting parameters results in a change of heat flows through the mushy zone and also in a change of its configuration. The growth conditions in mushy zone are essentially different and can not be described by the values of the temperature gradient and cooling rate in any single point. The first part of the present paper describes the results of the theoretical study of growth conditions on the mushy zone boundaries - on the surfaces of solidus and liquidus.

Among the structural factors, which define the mechanical properties of a material, the grain structure shows an important feature. The columnar grains nucleated on the chill-plate change their transversal dimensions with increased distance from the chillplate. The phenomenon of grain structure spreading is observed in DS bucket for industrial gas turbine engines. In previous papers the authors [1-3] have suggested a theoretical model of grain spreading process in conditions of unidirectional heat transfer. The model is based on the concept of mobility of grain boundaries during growth. It has shown that the increase of grain cross sections obeys the law of normal kinetic. The second part of the paper describes data of columnar grain structure investigation of real buckets and blades. To analyze the obtained experimental data, the geometrical theory of interface motion was used.

\section{The mushy zone of casting}

As is known, during the directional solidification (DS) of superalloys, macro- and microstructure forms in the mushy zone where dendrites of the solid phase and the melt coexist. The mushy zone boundaries are the isothermal surfaces $T_{S}=$ const and $T_{L}=$ const. 
The mushy zone extent along the direction of the solidification can be from several millimeters to several centimeters depending on a temperature interval of alloy crystallization $\left(\Delta T=T_{L}-T_{S}\right)$ and thermal gradient. The thermal gradient varies from point to point of the isothermal surfaces $T_{L}=$ const and $T_{S}=$ const and the values of thermal gradient can essentially be different.

The scheme of heat flows for the mushy zone is shown in Figure 1.

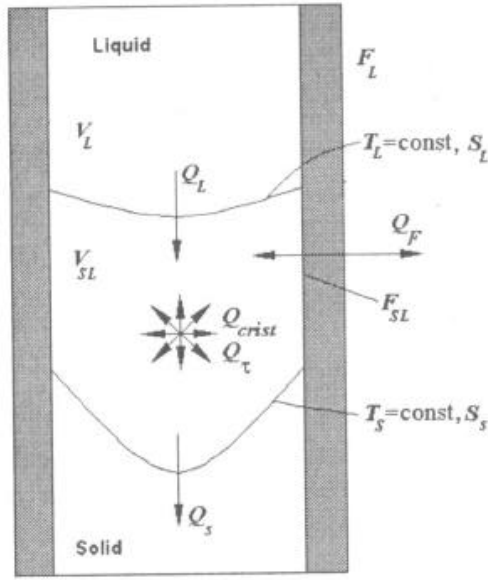

Figure 1: The scheme of thermal flows in the mushy zone of DS casting

The heat balance equation for a mushy zone can be written as:

$$
Q_{S}=Q_{L}+Q_{\text {crist }}+Q_{\mathrm{r}}+Q_{F} \text {, }
$$

where $Q_{S}$ is the quantity of heat output from the mushy zone through the surface $T_{S}=$ const; $Q_{L}$ is the quantity of heat input to the mushy zone through the surface $T_{L}=$ const; $Q_{\text {crist }}$ is the crystallization heat; $Q_{\tau}$ is the quantity of heat output from the mushy zone as a result of metal cooling; $Q_{F}$ is the quantity of heat input to (or output from) the mushy zone through the mould surface.

$$
\begin{aligned}
& Q_{S}=\lambda_{m}\left(T_{S}\right) \int_{S}|\operatorname{grad} T| d F=\lambda_{S} G_{S} S_{S} \\
& Q_{L}=\lambda_{m}\left(T_{L}\right) \int_{S_{t}}|\operatorname{grad} T| d F=\lambda_{L} G_{L} S_{L}
\end{aligned}
$$

where $\lambda_{m}(T)$ is the thermal conductivity of the metal depending on temperature; $G_{L}, G_{S}$ are the average thermal gradients at the surfaces of $T_{L}$ and $T_{S}=$ const; $S_{L}, S_{S}$ are the areas of the surfaces $T_{L}$ and $T_{S}=$ const; $\lambda_{L}, \lambda_{S}$ are the thermal conductivity at the temperature $T_{L}$ and $T_{S}$

When the mushy zone moves with a steady velocity $w$ and its configuration remains constant while moving

$$
Q_{\text {rist }}=\rho_{L} L w S_{r}
$$

where $\rho_{L}$ is the melt density at the temperature $T_{L} ; L$ is the laten heat; $S_{S}$ is the transversal section area of the casting, and

$$
Q_{\tau}=w \int_{V_{s c}} \rho_{m}(T) C_{m}(T)|\operatorname{grad} T| d V
$$

where $\rho_{m}(T)$ is the density of alloy; $C_{m}(T)$ is the specific heat of alloy; $V_{S L}$ is the volume of the mushy zone.

The influence of last term of the equation (1) depends on th mushy zone position in the heater.

$$
Q_{i}=\int_{F_{\mathrm{u}}} q_{f} d F
$$

where $q_{f}$ is the heat flow through the lateral surface of the casting $F_{S L}$ is the lateral surface of the casting in the mushy zone (se Figure.1). Thus, the heat balance equation for the mushy zone (1 becomes

$$
\begin{aligned}
\lambda_{S} G_{S} S_{S}=\lambda_{L} G_{L} S_{L}+w & {\left[\rho_{L} L S_{r}+\int_{V_{s L}} \rho_{m}(T) C_{m}(T)|\operatorname{grad} T| d V\right] } \\
& +\int_{F_{s}} q_{f} d F
\end{aligned}
$$

The equation for a heat flow through the isothermal surface of $T_{L}=$ const can be written similarly equation (7):

$$
\lambda_{L} G_{L} S_{L}=\int_{V_{\curlywedge}} \rho_{m}(T) C_{m}(T) \frac{\partial T}{\partial \tau} d V+\int_{F_{L}} q_{f} d F,
$$

where $F_{L}$ is the surface of casting above the isothermal surfac $T_{L}=$ const.

The last terms of the equations (7) and (8) determine the quanti of heat transferred through the casting surface as a result of a he exchange with a furnace. They depend on the mushy zor position in the heater and the heater temperature. Thus, the la term of equation (7) can change its sign depending on the must zone position into the hot or cold zone of the furnace. The secor term of the equation (7) contains the solidification rate $w$, which proportional to the mould withdrawal rate. The first term equation (8) contains the cooling rate of the melt $\partial T / \partial \tau$, whis also is proportional to the withdrawal rate.

Average thermal gradients $G_{L}$ and $G_{S}$ are proportional to tl quantity of heat transferred through the corresponding isotherm surface in unit time. Therefore it is necessary to increase he flows through the mushy zone to increase the thermal gradient. On the other hand, $G_{L}$ and $G_{S}$ are inversely proportional to t areas of the isothermal surfaces. The increase of heat flows $w$ entail a displacement of the mushy zone in a heater, a flexure of configuration and, hence, an increase of the isothermal surfa solidus (liquidus) area $S_{L}, S_{S}$. In this case a change of the casti conditions will entail a reduction of the temperature gradients. Thus, the equations (7) and (8) demonstrate a compl dependence of the thermal gradients $G_{L}$ and $G_{S}$ from major casti 
process parameters - the mould withdrawal rate and the temperature of the heater.

\section{Mathematical model}

To define forming conditions of the casting structure, computing experiments have been conducted. The software CASTDS ${ }^{2 D}$ was used to simulate the thermal conditions of the Bridgman-type DS process. The effect of the heater temperature, the withdrawal rate and the mould thermal conductivity on the thermal gradient, solidification rate and the melt cooling rate in the mushy zone have been studied.

Mathematical basis of the CASTDS $^{2 D}$ model is a numerical solution of the unsteady heat conduction equation for the cylindrical mould.

In the CASTDS ${ }^{2 \mathrm{D}}$ software the furnace consists of a cylindrical heater, a cylindrical side cooler and a chill plate. Thermal properties of mould material and metal depend on the temperature.

The input data for the simulation are the temperature profiles along the furnace as a function of time, initial mould position and withdrawal rate, the pouring time point and the temperature of the metal poured into the mould.

Computing experiments consisted of the mould heating process simulation from a room temperalure up to the pouring time point, the imitation of pouring process, calculation of the temperature distribution in the mould and solidifying metal during their moving out of heater.

The following factors have been taken into consideration:

- the radiation heat transfer from the surfaces of the heater to the surface of the ceramic mould and the meit surface;

- the radiation heat transfer between the ceramic mould surface and upper and lower surfaces of the baffle between hot and cold zones;

- the radiation heat transfer from the surface of the ceramic mould to the surfaces of the vacuum chamber and the side cooler;

- the convection and radiation heat transfer from the mould and metal to the chill plate. The chill plate temperature is established as a result of simulation of this heat process.

It was supposed that all elements of the furnace hot zone were made of graphite and their emissivity factor is equal to 1 . The emissivity factors of ceramics and a melt in the mould are much less than 1. However the surfaces of the melt and the cylindrical mould are convex, so that it can be excluded from the account multiple reflections in the radiation heat transfer simulation.

The computing experiments were carried out for the cylindrical form casting with a diametcr of $22 \mathrm{~mm}$ and a length of $450 \mathrm{~mm}$. In Figures $2 \mathrm{a}$ and $2 \mathrm{~b}$ the typical curves of thermal gradients $G_{L}$ and $G_{S}$, solidification rates $w_{L}$ and $w_{S}$ of the solidus and the liquidus isotherms are shown depending on their position in casting. In Figure $2 \mathrm{~d}$ the position of isotherms $T_{L}$ and $T_{S}$ in casting depending on its position in a heater is shown. All results relate to an axis of casting.

It is possible to assume that the conditions in the mushy zone in initial time point mainly depend on metal pouring temperature and temperature of the heater. In a mushy zone the conditions are established in compliance with a given regime of the directional solidification during the initial stage of solidification. The mushy zone displaces in the heater to the position appropriated to the given withdrawal rate and the heater temperature. At this stage of solidification the velocity of the isotherms $T_{L}$ and $T_{S}$ movement can differ strongly from the withdrawal rate.

As shown in Figure 2, the initial stage is completed when it has reached stable conditions of solidification at constant values of the thermal gradient and the solidification rate. The time required to reach a steady state, and the duration of steady state solidification depends on the casting parameters and geometry of the casting. The final stage of a solidification of casting takes place, when an influence of the top of casting becomes significant. At high heater temperature the additional heat of metal through its free surface results to an increase of thermal gradient and displacement of the mushy zone in the direction of the cooler. At low heater temperature, insufficicntly intensive heat of metal results in a displacement of the mushy zone in a direction of the heater and decrease of thermal gradient.
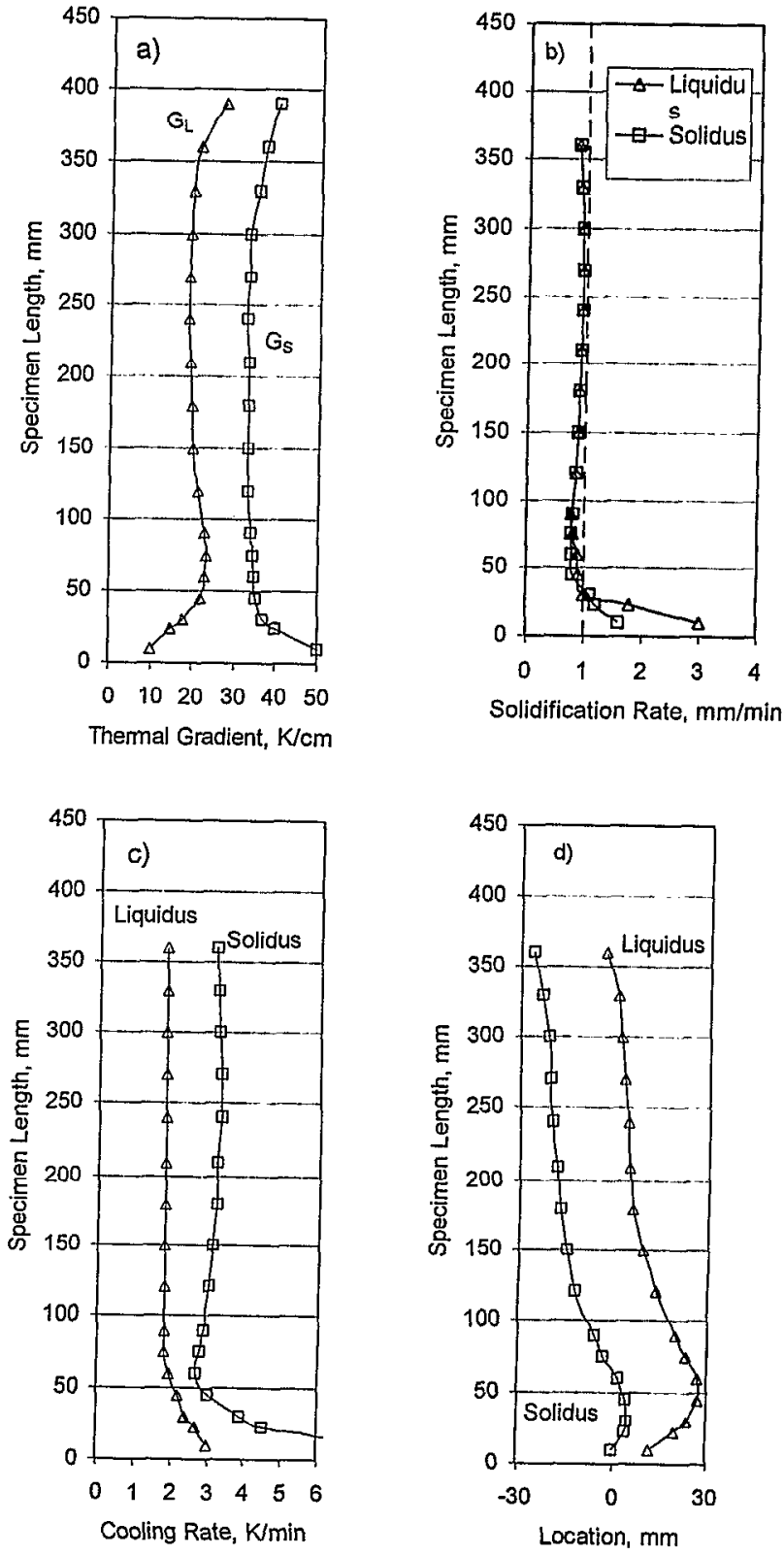

Figure 2: The typical calculated curves of the thermal gradient (a), solidification ratc (b), cooling rate (c) and the mushy zone position (d) for simulated directional solidification process. 
The results are related to a steady slate regime of the solidification. The values $G_{L}$ and $w_{L}$ at the isotherm $T_{L}=$ const and its position $x_{L}$ were determined at the time point, when casting axis temperature of section $x=240 \mathrm{~mm} T=T_{L}$. The values $G_{S}, w_{S}$ and $x_{S}$, were determined at the same time point in the section, in which the casting axis temperature was $T=T_{S}$.

\section{Influence of the withdrawal rate.}

As shown in equations (7) and (8), there are additional sources of heat at a high rate of the directional solidification, which should supply higher temperature gradient in comparison with that of quasistationary conditions. However, when the withdrawal ratc is increased, the temperature gradient grows at the solidus only (Figure 3).

At the low withdrawal rate, about several millimeters in an hour, the contribution of the non-stationary terms of the right part of the equation (7) is insignificant. The relationship between $G_{S}$ and $G_{L}$ is determined as the relationship of surfaces $S_{S}$ and $S_{L}$.
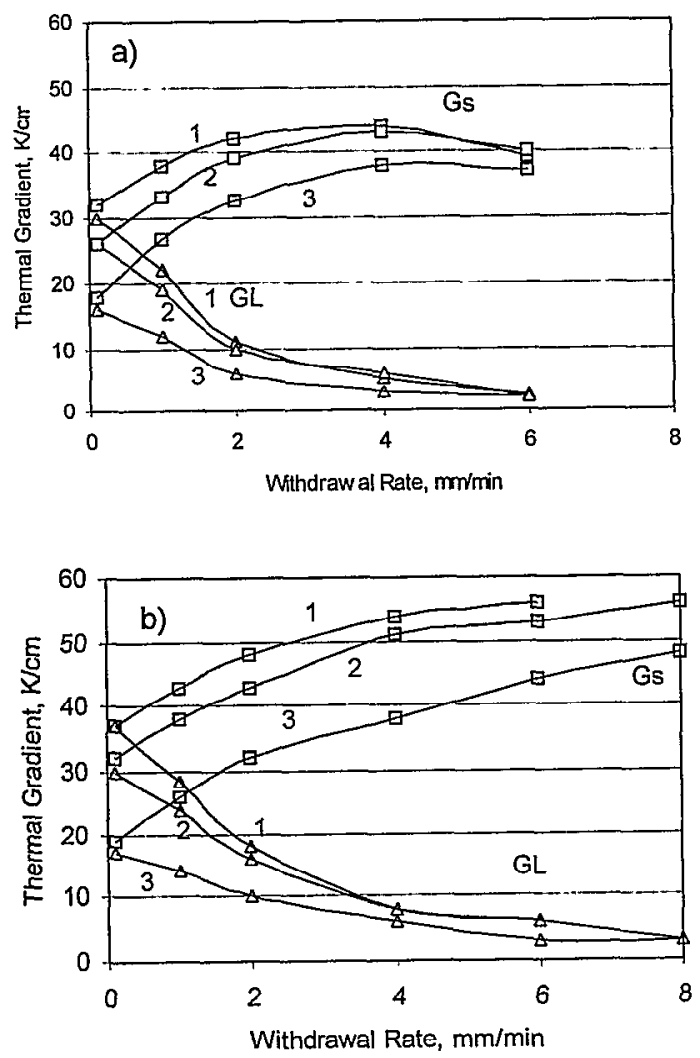

Figure 3: Calculated temperature gradient at the isothermal surfaces of solidus $G_{S}$ and liquidus $G_{L}$ depending on the withdrawal rate and heater temperature. (a) - low thermal conductivity of the mould $\lambda_{f}=2.2 W /(\mathrm{mK})$; (b) - high thermal conductivity of the mould $\lambda_{f}=20.2 \mathrm{~W} /(\mathrm{mK})$. I - the heater temperature $T_{h}=1700^{\circ} \mathrm{C} ; 2$ - the heater temperature $T_{h}=1600^{\circ} \mathrm{C} ; 3$ - the heater temperature $T_{h}=1500^{\circ} \mathrm{C}$.

With increased withdrawal rate, the liberation of the latent heat results in heating of metal in the mushy zone. It reduces $G_{L}$ and

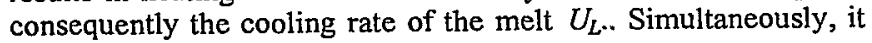

results in an increase of the amount of heat left from the mushy zone through the solidus surface and an increase of the meltcooling rate at the solidus $U_{S}$.

A change of the relationship between $G_{S}$ and $G_{L}$ due to a change of the mushy zone configuration, also results in a change of the relationship between $G_{S}$ and $G_{L}$. The $G_{S}$ growth with an increase of the withdrawal rate is decelerated with a displacement of the mushy zone in the side cooler area. Consequently, a sign of the last term is changed in equations (7) and (8).

On the one hand, the mushy zone cooling through the mould side walls reduces a part of the thermal energy took off through the surface $S_{S}$, and on the other hand, it results in an increase of the surface $S_{S}$ area.

The influence of the withdrawal rate on conditions in the mushy zone will vary depending on the furnace design, thermal properties of the mould and casting geometry.
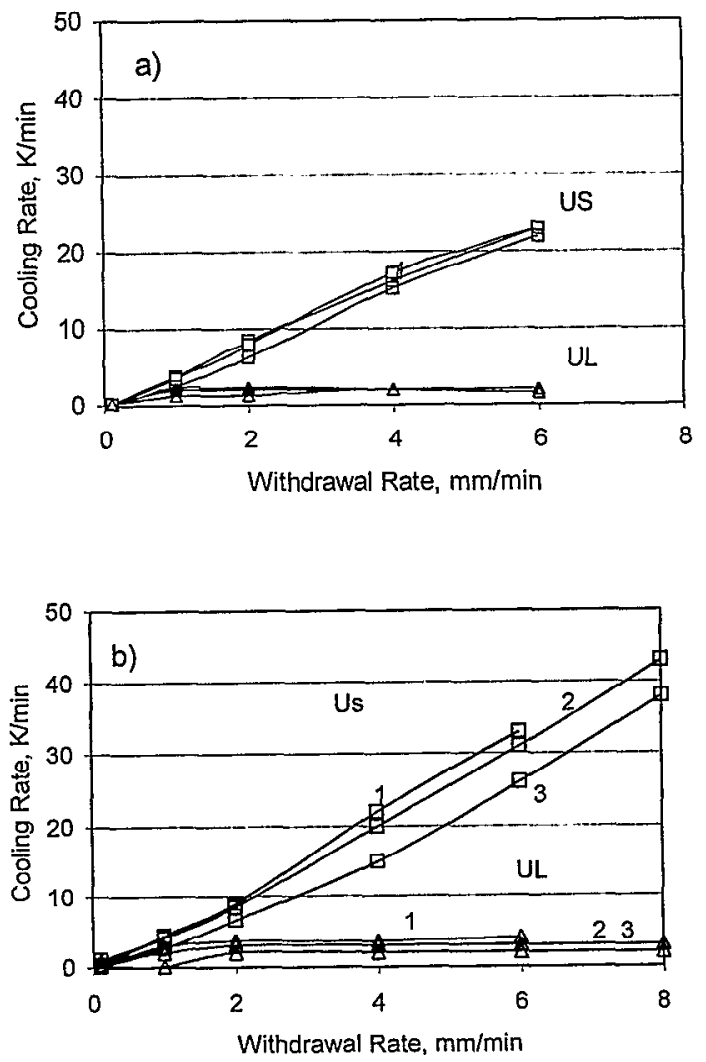

Figure 4: Calculated melt cooling rate at the isothermal surfaces of solidus $U_{S}$ and liquidus $U_{L}$ depending on the withdrawal rate and heater temperature. (a) - low thermal conductivity of the mould $\lambda_{j}=2.2 \mathrm{~W} /(\mathrm{mK})$; (b) - high thermal conductivity of the mould $\lambda_{j}=20.2 \mathrm{~W} /(\mathrm{mK}) .1$ - the heater temperature $T_{h}=1700^{\circ} \mathrm{C} ; 2$ - the heater temperature $T_{h}=1600^{\circ} \mathrm{C} ; 3$ - the heater temperature $T_{h}=1500^{\circ} \mathrm{C}$

\section{Influence of the thermal conductivity of the mould.}

The results, shown in Figures 3-6, enable are to estimate a change of the thermal conditions in the mushy zone in a practical significant range of the mould thermal resistance. The calculations 
are presented for the mould thickness of $14 \mathrm{~mm}$ and the thermal conductivity of ceramics $\lambda_{f}=2.2 \mathrm{~W} /(\mathrm{mK})$ and $\lambda_{f}=20.2 \mathrm{~W} /(\mathrm{mK})$.

The tenfold increase of the thermal conductivity results in some increase of the temperature gradient and the cooling rate in the mushy zone. The greatest growth (up to $30 \%$ ) of this value is observed at the high heater temperature (Figure 6) and at the high withdrawal rate (Figures 3 and 4).

The influence of thermal conductivity of the mould on the mushy zone position in the furnace is quite significant. The increase of the thermal conductivity has not changed the sensitivity of the mushy zone position to the temperature of a heater, but it has made the considerably less sensitive to the withdrawal rate.

According to the Figure 5a, for a mould with the thermal conductivity $\lambda_{f}=2.2 \mathrm{~W} / \mathrm{m} / \mathrm{K}$, the withdrawal rate $5-6 \mathrm{~mm} / \mathrm{min}$ fits to a very low mushy zone position practically at the exit of the side cooler. It is possible to assume that the further increase of the withdrawal rate will reduce to the violation of the unidirectional heat transfer, i.e. to the equiaxed solidification.
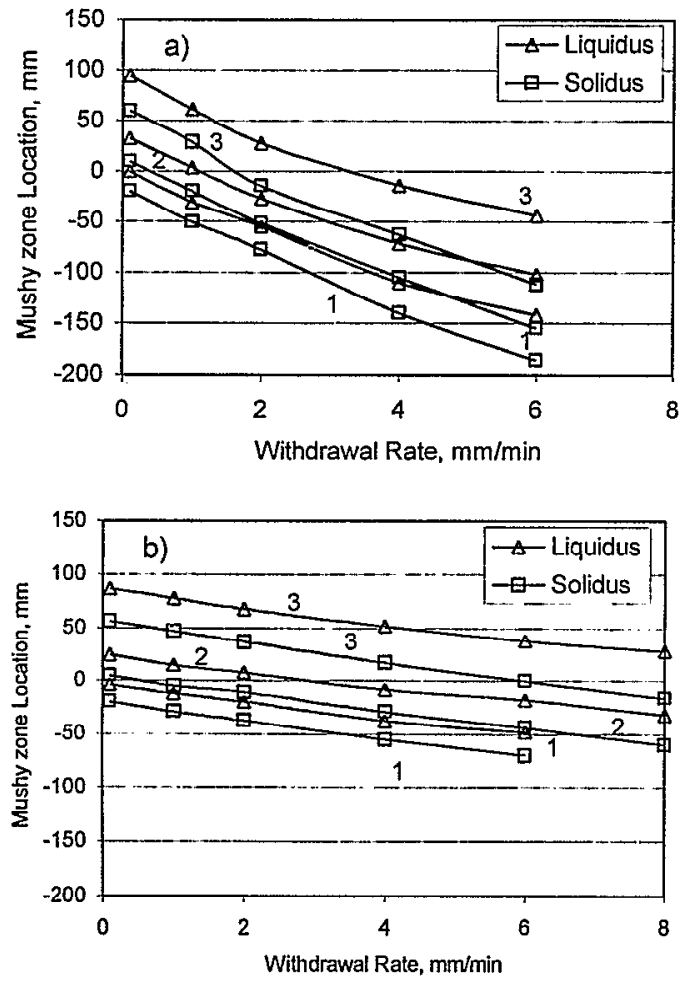

Figure 5: Calculated position of the isothermal liquidus and solidus surfaces depending on the withdrawal rate and heater temperature. (a) low thermal conductivity of the mould $\lambda_{f}=2.2 \mathrm{~W} /(\mathrm{mK})$; (b) high thermal conductivity of the mould $\lambda_{f}=20.2 \mathrm{~W} /(\mathrm{mK}) .1$ - the heater temperature $T_{h}=1700^{\circ} \mathrm{C} ; 2$ - the heater temperature $T_{h}=1600^{\circ} \mathrm{C} ; 3$ - the heater temperature $T_{h}=1500^{\circ} \mathrm{C}$.

In case of solidification in a high thermal conductivity mould, an increase of the withdrawal rate has a smaller effect on the mushy zone position. The application of higher heat conductive ceramics allows to increase the withdrawal rate at the invariable position of the mushy zone in the furnace.

\section{Materials and Experimental}

Casting of buckets was undertaken with a commercial scale vacuum induction furnace. The Bridgmen's method was used as a base of casting technology. The furnace has the following technical parameters: weight of liquid metal is about $20 \mathrm{~kg}$, operating height of the solidification unit is $400 \mathrm{~mm}$, three- zones heater with individual regulation, maximal operating temperature of heater is $1650^{\circ} \mathrm{C}$ and diameter of chill-plate is $210 \mathrm{~mm}$.

The withdrawal mechanism allows to regulate velocity of mould moving from $0.01 \mathrm{~mm} / \mathrm{min}$ up to $20 \mathrm{~mm} / \mathrm{min}$. The special transitions provide the possibility of using 20 and more thermocouples to monitor the tempcrature during casting.
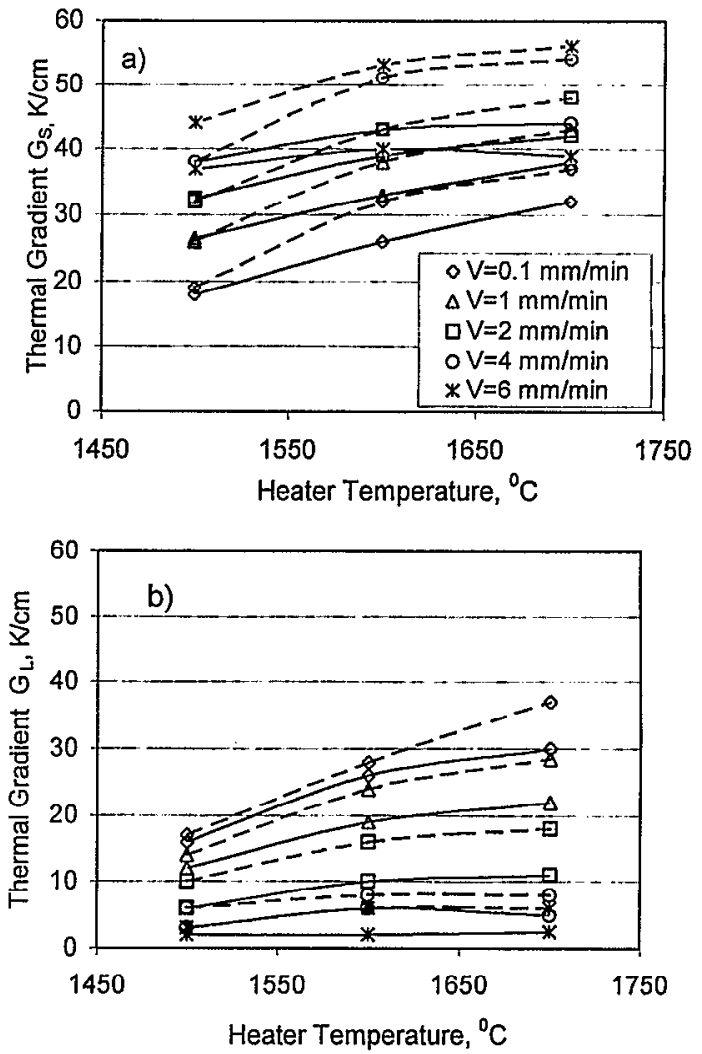

Figure 6: Calculated temperature gradient at the isothermal surfaces of solidus $G_{S}$ (a) and liquidus $G_{L}$ (b) depending on the heater temperature and the mould thermal conductivity. Solid lines mean low thermal conductivity of the mould $\lambda_{j}=2.2 \mathrm{~W} /(\mathrm{mK})$; Dashed lines mean high thermal conductivity of the mould $\lambda_{j}=20.2 \mathrm{~W} /(\mathrm{mK})$.

To investigate alloy"s composition effect, IN738LC (WILLAN Co., UK) and B1914 were used. The chemical compositions of these alloys are presented in Table 1.

Growth of the structure of blades and buckets was undertaken at the furnace temperatures of $1450-1500^{\circ} \mathrm{C}$ and under the withdrawal rate of $2.5-6.0 \mathrm{~mm} / \mathrm{min}$.

Transverse sections of the top, middle and transition to platform for DS-airfoil were examined by metallographic techniques. 
TABLE I. Composition of the Alloys (mass \%)

\begin{tabular}{ccc}
\hline Element & B 1914 & IN 738LC \\
\hline $\mathrm{Cr}$ & 10.10 & 15.78 \\
$\mathrm{Co}$ & 9.80 & 8.42 \\
$\mathrm{Mo}$ & 3.10 & 1.70 \\
$\mathrm{Ti}$ & 5.27 & 3.37 \\
$\mathrm{Al}$ & 5.58 & 3.32 \\
$\mathrm{C}$ & 0.001 & 0.10 \\
$\mathrm{Ta}$ & - & 1.70 \\
$\mathrm{~W}$ & - & 2.55 \\
$\mathrm{~B}$ & 0.11 & 0.008 \\
$\mathrm{Fe}$ & 0.11 & $10 \mathrm{ppm}$ \\
$\mathrm{Nb}$ & - & 0.70 \\
$\mathrm{Ni}$ & $\mathrm{Bal}$ & Bal. \\
\hline
\end{tabular}

The OMNIMET ${ }^{B}$ I" BUEHLER Image Analyze System was used to examine the grain sizes. The Standards Test Method for Determining Average Grain Size (E 1382-91 and E1181) was used. Each grain was measured in four different positions through the center of grain.



Figure 7: The distribution of the measured grain width in the two transversal sections of directionally solidified blades of IN738LC.

\section{Grain Size Measurement}

The measurement data of grains from castings with columnar structure are presented on Figures $7-10$ in the histogram fashion. The analysis of results shows that the grain spreading takes place in all cases of structure growth. The value of spreading is not identical for the studied alloys. The grains in castings of B1914 alloy spread more intensively.

In small size castings (total length is about $80 \mathrm{~mm}$, thickness of airfoil is about $2-4 \mathrm{~mm}$, weight is about $0.1-03 \mathrm{~kg}$ ) the columnar structure is characterized with small sized grains cross section at the entrance into the airfoil. The dimensions of majority grains do not exceed $1.2 \mathrm{~mm}$ with the maximum distribution curve at $0.6 \mathrm{~mm}$ for IN738LC and $2.0 \mathrm{~mm}$ with maximum of $0.8-1.0 \mathrm{~mm}$ for B1914 alloys.

The estimation of grains sizes in the sections, placed at the distance $30 \mathrm{~mm}$ showed displacement of maximum of distribution curves to the right till $1,3 \mathrm{~mm}$ for IN738LC and $1,5-1,7 \mathrm{~mm}$ for B1914.

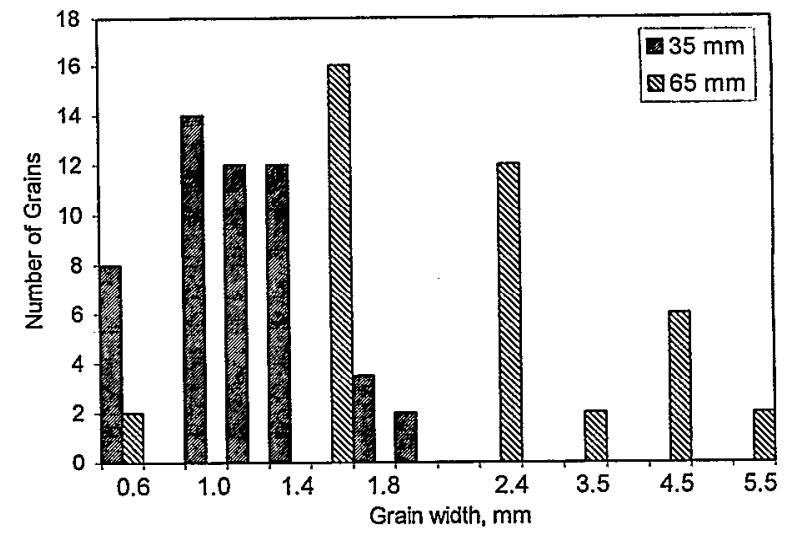

Figure 8: The distribution of measured grain width in two transversal sections of directionally solidified blades of B1914 alloys.



Figure 9: Grain size distribution versus distance from the chillplate for the DS bucket of IN738LC alloy

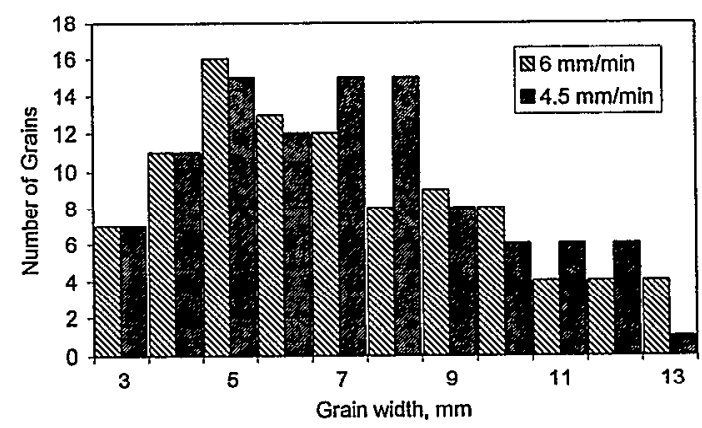

Figure 10 shows the measurement data of grains size in the cross section of the root of buckets solidified at the two different withdrawal rates. 
The same tendency was noticed at the estimation of the grain dimensions in buckets of IN738LC. The grain structure loses the uniformity with the distance from chill-plate. The structure of the cross section at the distance of $35 \mathrm{~mm}$ from chill-plate is rather uniform. The maximum of the distribution curve moves also to the right, (to the side of bigger grain dimensions $-4.0 \mathrm{~mm}$ at the dislance $155 \mathrm{~mm}$ and $5.5 \mathrm{~mm}$ at $235 \mathrm{~mm}$ respectively). It is necessary to mark the appearance of the very large grains with a cross section of 10-16 mm though in small quantities - no more than $4 \%$ of the total number of grains (Figure 9 ).

\section{$\underline{\text { Discussion }}$}

As shown in Figures $7-10$, the transverse size of columnar grains increases with the increase of a distance from the examined section to the chill-plate. To describe the growth of the columnar grain width quantitatively, the following physical model can be considered. All grains have nucleated on the chill plate and grow by the mushy zone movement. The average transverse grain size increases with the distance from the chill-plate. The increase of the transversal size of grains reached section $X$ has taken place at the expense of their neighbors during competitive growth.

According to [1] the grain width grows under the normal growth law, independent of the growth morphology and can be described as following:

$$
R^{m}=R_{u}^{m}+M \tau
$$

where $\tau$ is the growing time, $R$ is the average transverse grain size at the time $\tau, R_{0}$ is the average transverse grain size at time $\tau=0$; $M$ is mobility of the grain boundary; $m$ is the grain growth exponent.

It is obvious to assume that the transverse grain growth is possible only into the mushy zone. Then the time of the grain growth will be determined with the mushy zone moving velocity and its position in casting. Assuming that the mushy zone moving velocity is equal to the withdrawal rate, then the equation (9) can be rewritten as

$$
R^{m}=R_{\mathrm{o}}^{m}+M \frac{X}{V}
$$

where $X$ is the mushy zone position at time $\tau, V$ is the withdrawal rate.

As the initial size of grain nucleated on the chill-plate $R>>R_{0}$, the equation (10) can be transformed to

$$
R=\left(M \frac{X}{V}\right)^{1 / m}
$$

Thus, the experimentally observable increase of the transverse size of columnar grains in the directional solidification can be described by

$$
R=A\left(\frac{X}{V}\right)^{\prime \prime}
$$

where $A$ and $n$ are coefficient and exponent defined from experimental data.

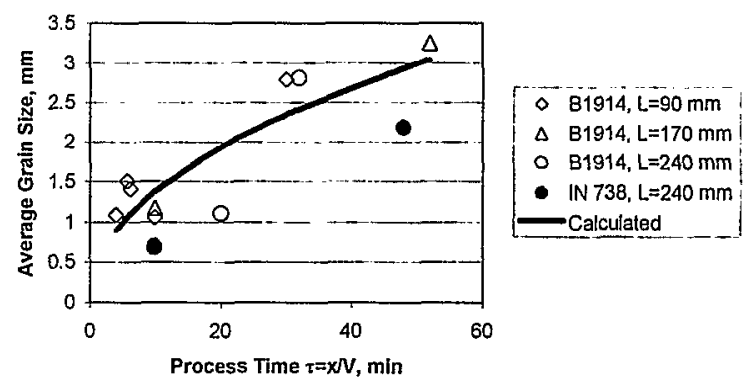

Figure 11: Comparison of calculated and measured columnar grain size of DS blades and buckets. $\mathrm{L}$ is the length of casting part.

Figure 11 shows the measured average transverse size of columnar grains in different cross-sections of blades and buckets with different withdrawal rate. The received data of B1914 alloy are approximated by the equation,

$$
R=0.46\left(\frac{X}{V}\right)^{\frac{1}{2}}
$$

which is in good agreement with experimental data. In equation (13) exponent $n=1 / 2$ and hence in the equations (9) (11) exponent $m=2$. This value does not contradict the conventional notion when $2 \leq m \leq 3$ [4]. In addition, Wagner reported that $m=3$ for the diffusion controlled grain growth and $m=2$ for the interfacial-reaction controlled growth. However, it is not easy to debate the mechanism of interaction of grains at the competitive growth on the castings with a variable cross-section area - real turbine blades. The model of the competitive growth of columnar grains, suggested in [5] and [6], explains transversal growth of the grains at the expense of neighbors by the leading growth of the dendrites with preferable crystallographic orientation. Based on this model, it is possible to assume the process of transversal growth of columnar grains is controllcd more dominantly by an interaction of the dendritic skeleton of grains than the diffusion processes in the mushy zone. Thereforc, the exponent $m=2$ obtained from equation (13) seems more realistic. Therefore, we suggest in this work that the interfacial reaction process rather than the diffusion process control the grain widening in DS casting.

\section{Conclusions}

Modeling of different conditions of Bridgman's solidification allows one to investigate the influence of the technical casting parameters such as withdrawal rate, heater temperature and thermal conductivity of mould on the value of thermal gradient and cooling rate at the liquidus and solidus surfaces and on the mushy zone position.

The columnar grain structure of blades and buckets of B1914 and IN 738LC alloys solidified by Bridgman's process was investigated. It has been shown that the average transversal size of the columnar grains increases along the casting. The analysis of 
the obtained experimental data, based on the geometrical theory of interface motion, has allowed one to receive an approximate dependence of the columnar grains transversal size on the distance up to their nucleation site The result of approximation is in agreement with the experimental data.

\section{$\underline{\text { References }}$}

1. E. A. Holm, N. Zacharopoulos and D. J. Srolovitz, "Nonuniform and Directional Grain Growth Caused by Grain Boundary Mobility Variations", Acta Mater., 46 (3) (1998), 953964.

2. J. E. Taylor, J. W. Cahn and C. A. Handwerker, "Geometric Models of Crystal Growth", Acta Metall. Mater., 40 (7) (1992), 1443-1474.

3. B. Szupar and R. W. Smith, "Monte Carlo Simulation of Solidification Processes; Porosity", Canadian Metallurgical Quarterly, 35 (1996) 299.

4. V. Tikare and J. D. Cawley, "Numerical simulation of grain growth in liquid phase sintercd materials", Acta Mater., 46 (4) 1998, 1343-1356.

5. M. Rapaz and Ch.-A. Gandin, "Probabilistic modelling of microstructure formation in solidification processes", Acta Mater., 41 (2) (1993), 345-360.

6. V. Monastyrsky and E. Kachanov. "The model of the competitive growth of columnar grain of multi-component alloy". Solidification and modeling. Transaction of the IV All Russia conference on problem on alloys solidification and computer modelling, Igevsk, October (1990), 10-24 (in Russian). 